www.nature.com/ja

\title{
The psychotrimine natural products have antibacterial activity against Gram-positive bacteria and act via membrane disruption
}

\author{
Mark A Schallenberger, Timothy Newhouse, Phil S Baran and Floyd E Romesberg
}

The Journal of Antibiotics (2010) 63, 685-687; doi:10.1038/ja.2010.107; published online 6 October 2010

Keywords: antibiotic; membrane damage; natural product; plant indole alkaloids

\begin{abstract}
Antibiotic therapy places intense selection pressure on bacteria that inevitably leads to the emergence of resistance, ${ }^{1}$ and thus the need to continually identify and develop new antibiotics. Secondary metabolite natural products have been, and seem likely to continue to be, the best source of antibacterial agents, providing the vast majority of therapeutics actually used in the clinic. ${ }^{2}$ As a result, it is of great interest to examine newly identified secondary metabolites for their potential antibacterial activity. There is a growing appreciation that plants provide a unique source of diverse secondary metabolites with potentially important biological activities, with one of the most promising classes being the indole alkaloids. ${ }^{3}$ One such indole alkaloid natural product is psychotrimine (1, Figure 1), which was isolated in 2004 from leaves of the South American shrub Psychotria rostrata, ${ }^{4}$ and has attracted considerable interest from the synthetic and medicinal chemistry communities owing to its unusual connectivity between tryptamine subunits. The peculiar N1-C3 linkage differs from the carbon-carbon linkages that are more typical of other polymeric indole alkaloids. ${ }^{5}$ Preliminary biological studies showed that psychotrimine has only mild antiproliferative activity against several colon and lung cancer cell lines. ${ }^{6}$ Although interest in the indole alkaloids has generally been focused on their anticancer, antiinflammatory and neurological activities, there is growing evidence that these compounds may provide promising scaffolds for antibiotic development. $^{3}$
\end{abstract}

With access to psychotrimine provided by the recently reported gram scale total synthesis, ${ }^{6}$ we sought to examine the potential antibacterial activity of this unique indole alkaloid. We first screened a variety of bacteria for sensitivity to psychotrimine according to CLSI guidelines. ${ }^{7}$ No activity was observed against Pseudomonas aeruginosa PA01 or Klebsiella pneumoniae ATCC 43816 up to $128 \mu \mathrm{g} \mathrm{ml}^{-1}$. We observed mild activity against Escherichia coli MG1655, inhibiting growth with an MIC of $128 \mu \mathrm{g} \mathrm{ml}^{-1}$. Polymyxin B nonapeptide does not sensitize $E$. coli to psychotrimine, suggesting that the absence of activity against Gram-negative bacteria is not the result of limited outer membrane penetration. In contrast, more significant activity against Gram-positive bacteria was observed. Psychotrimine is reasonably active against Bacillus subtilis 168, Streptococcus agalactiae $\mathrm{COH} 1$ and S. pyogenes 5448, inhibiting growth of each with an MIC of $16 \mu \mathrm{g} \mathrm{ml}^{-1}$, and moderately active against Staphylococcus epidermidis RP62A and S. aureus 8325, inhibiting growth with an MIC of 32 and $64 \mu \mathrm{g} \mathrm{ml}^{-1}$, respectively.

To further understand the Gram-positive activity of psychotrimine, we constructed kill curves for both S. aureus and B. subtilis. Log-phase bacteria $\left(1 \times 10^{7}\right.$ c.f.u. $)$ were inoculated into Mueller-Hinton media containing $2 \times$ MIC of psychotrimine and incubated with shaking at $37^{\circ} \mathrm{C}$. Aliquots were removed at various time points and appropriate dilutions were plated onto Mueller-Hinton agar to determine the number of viable cells. After only $3 \mathrm{~h}$, we observed a 300- and 1300fold decrease in viable cells for $S$. aureus and B. subtilis, respectively, indicating that psychotrimine has bactericidal activity (Figure 2a). Moreover, exposure of $S$. aureus to psychotrimine for only $40 \mathrm{~min}$ reduced the viable cell count $90 \%$ and reduced the OD590 of the culture $25 \%$, indicating bacteriolytic activity.

To begin to understand the mechanism by which psychotrimine kills Gram-positive bacteria, we attempted to isolate resistant mutants of B. subtilis and S. aureus. However, after plating $>5 \times 10^{9}$ c.f.u. on Mueller-Hinton agar plates containing $2 \times$ MIC psychotrimine, no viable colonies could be recovered. Moreover, serial passage of B. subtilis cells in sub-MIC concentrations of psychotrimine for 50 generations also failed to yield resistant colonies. These data suggest that resistant mutants, at least in B. subtilis and S. aureus, are extremely rare. Such low levels of resistance are common with a variety of mechanisms, including membrane disruption, which is also consistent with the positively charged lipophilic structure of psychotrimine. ${ }^{8}$

Most antibiotics that act via membrane disruption show synergistic activity when combined with other antibiotics that are limited by 


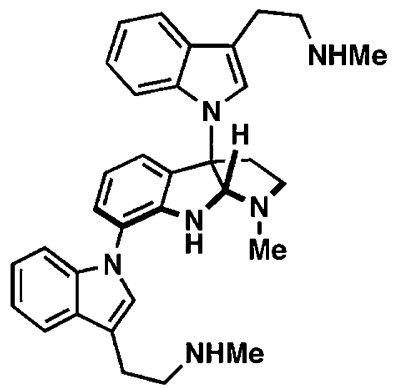

psychotrimine (1)

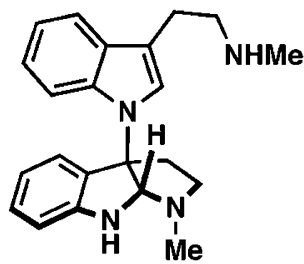

2

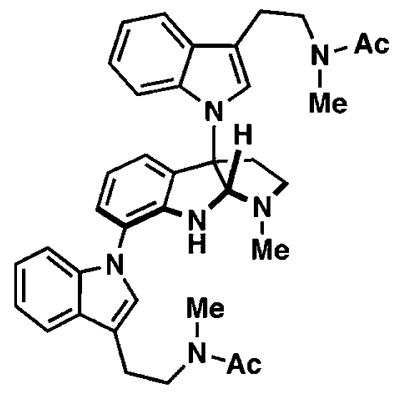

3

Figure 1 Structure of psychotrimine (1) and derivatives $\mathbf{2}$ and $\mathbf{3}$.
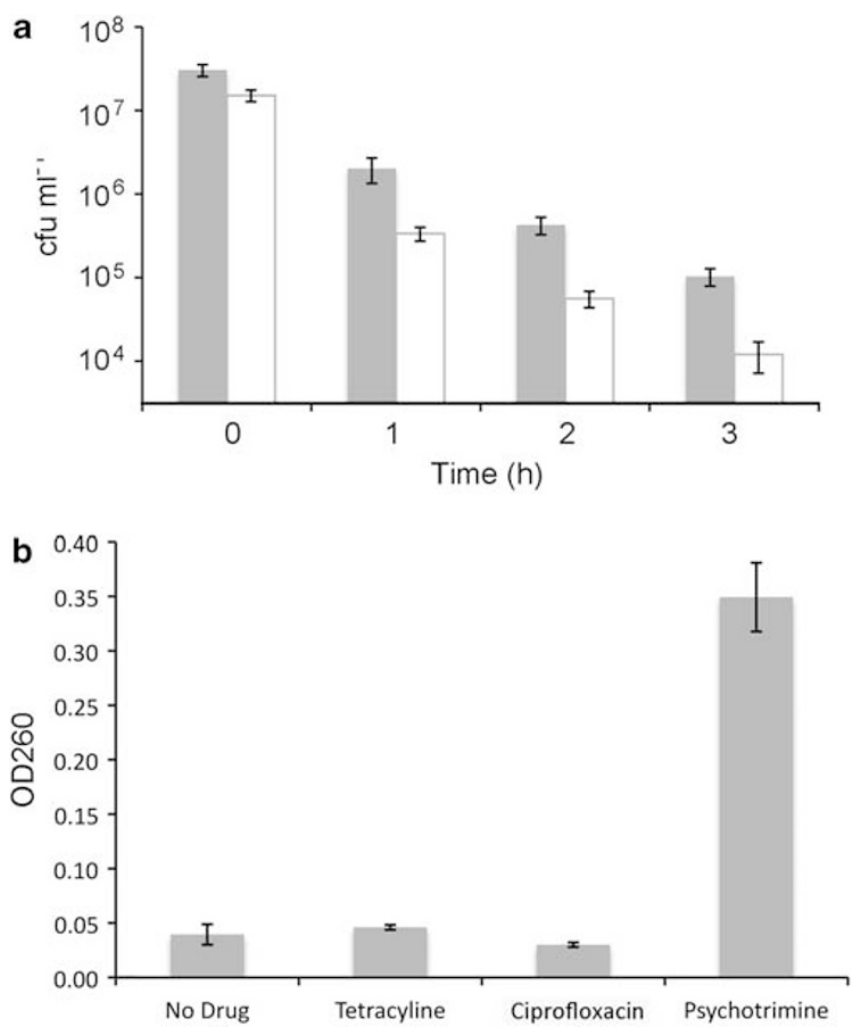

Figure 2 (a) Viable cell count following psychotrimine treatment of S. aureus (filled) and B. subtilis (open); and (b) absorption at $260 \mathrm{~nm}$ of supernatant from $S$. aureus cells following antibiotic treatment in phosphatebuffered saline (normalized for absorption of the antibiotic). Error bars represent standard deviation of at least three independent experiments.

membrane penetration, because they increase access to intracellular targets. ${ }^{9}$ Accordingly, we determined the MICs of rifampin and erythromycin against $E$. coli and $S$. aureus as a function of psychotrimine concentration. Rifampin and erythromycin are limited by membrane penetration with E. coli, but not with S. aureus. ${ }^{10}$ Psychotrimine showed substantial synergy with rifampin and erythromycin against E. coli, with $32 \mu \mathrm{g} \mathrm{ml}^{-1}(0.25 \times \mathrm{MIC})$ sensitizing E. coli cells 16-fold and 8-fold, respectively. In contrast, psychotrimine showed no interaction at any concentration with either drug against $S$. aureus. The observed synergy between rifampin or erythromycin and psychotrimine with E. coli but not $S$. aureus is further evidence for a membrane disruption-based mechanism of action.
We next used a more direct assay for psychotrimine-mediated membrane disruption, which relies on the detection of intracellular material released into the media following antibiotic treatment in osmotically stabilized buffer (Figure $2 \mathrm{~b}$ ). ${ }^{11}$ This assay was recently reported by O'Neill et al. ${ }^{12}$ to be more reliable than other assays used to detect membrane damage in S. aureus. S. aureus cells were grown to saturation and washed twice with phosphate-buffered saline to remove any extracellular material. The cells were collected by centrifugation and resuspended in buffer containing $1 \times \mathrm{MIC}$ of psychotrimine, tetracycline or ciprofloxacin. After $30 \mathrm{~min}$ of shaking at room temperature, the cells were removed by filtration and the OD260 of the supernatants was determined. As a control, a sample without any antibiotic was also prepared to account for any residual cell lysis and/ or protein secretion. The OD260 of the tetracycline- and ciprofloxacin-treated samples was $0.046 \pm 0.002$ and $0.030 \pm 0.002$, respectively, which are within error of that for the non-treated control $(0.039 \pm 0.009)$, consistent with little or no disruption of the bacterial membrane. ${ }^{12}$ In contrast, the OD260 of the supernatant from the psychotrimine-treated sample was $0.349 \pm 0.032$. This value is comparable with those previously reported for agents that strongly disrupt membranes, such as detergents and the natural product nisin. ${ }^{12}$ Because under the conditions employed there is little to no cell growth, it is unlikely that any cell lysis results from the inhibition of cellular targets. Thus, we conclude that psychotrimine acts by disrupting the cytoplasmic membrane of Gram-positive bacteria.

Two analogs (Figure 1) were synthesized to address whether the lipophilicity and positive charge of psychotrimine contribute to its antibacterial activity, as observed with other agents known to disrupt membranes. ${ }^{8}$ The C7-linked tryptamine unit of psychotrimine is removed in compound $\mathbf{2}$ and both secondary methylamines are acylated in compound 3. In both cases, at least one positive charge is removed. Information on the synthesis and characterization of the compounds is given in Supplementary Information. Both compounds $\mathbf{2}$ and $\mathbf{3}$ were found to have lost all activity against all of the bacteria tested (MIC $>128 \mu \mathrm{g} \mathrm{ml}^{-1}$ ). These data suggest that the presence of both secondary amines is critical for psychotrimine activity and is further consistent with a mechanism of action based on membrane disruption.

In conclusion, we have shown that psychotrimine disrupts the cytoplasmic membranes of both Gram-negative and Gram-positive bacteria and that in the latter case this results in reasonably potent antibacterial activity. The specific Gram-positive activity likely arises from unique aspects of their cytoplasmic membrane, such as charge or lipid composition, and similar activities have been observed with other plant indole alkaloids with structural homology to psychotrimine. ${ }^{13,14}$ This class of antimicrobials may be an important component of the 
plant defense system against the bacterial and perhaps fungal pathogens common in their environment. Because of the producing plants protective cell wall, these molecules can be produced at the high levels required for activity, and their mechanism of action makes them less susceptible to the evolution of resistance compared with more potent but target-specific antibiotics. ${ }^{15}$ Importantly, the biological insights generated in this study were only made possible from the large quantity of psychotrimine and its derivatives generated via total synthesis. Modifications of the synthesis should make possible similar analyses of other complex polymeric indole alkaloids and the further exploration of the potential biological activities of these interesting and novel secondary metabolites.

\section{ACKNOWLEDGEMENTS}

This work was supported by the National Institutes of Health (AI081126 to FER and CA134785 to PSB).

1 Clardy, J., Fischbach, M. A. \& Walsh, C. T. New antibiotics from bacterial natural products. Nat. Biotechnol. 24, 1541-1550 (2006).

2 Butler, M. S. \& Buss, A. D. Natural products-the future scaffolds for novel antibiotics? Biochem. Pharmacol. 71, 919-929 (2006).
3 Gul, W. \& Hamann, M. T. Indole alkaloid marine natural products: an established source of cancer drug leads with considerable promise for the control of parasitic, neurological and other diseases. Life Sci. 78, 442-453 (2005).

4 Takayama, H., Mori, I., Kitajima, M., Aimi, N. \& Lajis, N. H. New type of trimeric and pentameric indole alkaloids from Psychotria rostrata. Org. Lett. 6, 2945-2948 (2004).

5 Newhouse, T. \& Baran, P. S. Total synthesis of (+/-)-psychotrimine. J. Am. Chem. Soc 130, 10886-10887 (2008).

6 Newhouse, T., Lewis, C. A., Eastman, K. J. \& Baran, P. S. Scalable total syntheses of $\mathrm{N}$-linked tryptamine dimers by direct indole-aniline coupling: psychotrimine and kapakahines B and F. J. Am. Chem. Soc. 132, 7119-7137 (2010).

7 Clinical and Laboratory Standards Institute. Methods for Dilution Antimicrobial Susceptibility Tests for Bacteria that Grow Aerobically, CLSI: Wayne, PA, USA, (2006).

8 Payne, D. J., Gwynn, M. N., Holmes, D. J. \& Pompliano, D. L. Drugs for bad bugs: confronting the challenges of antibacterial discovery. Nat. Rev. Drug Discov. 6, 29-40 (2007).

9 Nikaido, H. \& Vaara, M. Molecular basis of bacterial outer membrane permeability. Microbiol. Rev. 49, 1-32 (1985).

10 Vaara, M. \& Vaara, T. Sensitization of Gram-negative bacteria to antibiotics and complement by a nontoxic oligopeptide. Nature. 303, 526-528 (1983)

11 Carson, C. F., Mee, B. J. \& Riley, T. V. Mechanism of action of Melaleuca alternifolia (tea tree) oil on Staphylococcus aureus determined by time-kill, lysis, leakage, and salt tolerance assays and electron microscopy. Antimicrob. Agents Chemother. 46, 1914-1920 (2002).

12 O'Neill, A. J., Miller, K., Oliva, B. \& Chopra, I. Comparison of assays for detection of agents causing membrane damage in Staphylococcus aureus. J. Antimicrob. Chemother. 54, 1127-1129 (2004).

13 Gibbons, S. Anti-staphylococcal plant natural products. Nat. Prod. Rep. 21, 263-277 (2004).

14 Mahmud, Z., Musa, M., Ismail, N. \& Lajis, N. H. Cytotoxic and bacteriocidal activities of Psychotria rostrata. Int. J. Pharmacog. 31, 142-146 (1993)

15 Lewis, K. \& Ausubel, F. M. Prospects for plant-derived antibacterials. Nat. Biotechnol. 24, 1504-1507 (2006).

Supplementary Information accompanies the paper on The Journal of Antibiotics website (http://www.nature.com/ja) 